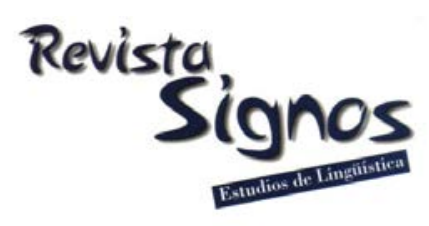

\title{
Panorama histórico y contrastivo de los estudios sobre lectura y escritura en educación superior publicados en América Latina
}

\section{A historical and contrastive account of higher education reading and writing studies published in Latin America}

\author{
Federico Navarro \\ UNIVERSIDAD DE BUENOS AIRES \\ CONICET \\ ARGENTINA \\ UNIVERSIDAD DE CHILE \\ CHILE \\ fnavarro@conicet.gov.ar
}

\author{
Natalia Ávila Reyes \\ PONTIFICIA UNIVERSIDAD CATÓLICA \\ DE CHILE \\ CHILE \\ naavila@uc.cl
}

\author{
Mónica Tapia-Ladino \\ UNIVERSIDAD CATÓLICA DE LA \\ SANTÍSIMA CONCEPCIÓN \\ CHILE \\ mtapia@ucsc.cl
}

\section{Vera L. L. Cristovão \\ UNIVERSIDADE ESTADUAL DE LONDRINA BRASIL \\ veraluciacristovao@gmail.com}

\author{
Maria Ester W. \\ Moritz \\ UNIVERSIDADE FEDERAL DE SANTA \\ CATARINA \\ BRASIL \\ nicamoritz@yahoo.com

\section{Charles Bazerman \\ UNIVERSITY OF CALIFORNIA SANTA BARBARA \\ USA \\ bazerman@education.ucsb.edu}
Elizabeth Narváez
Cardona
UNIVERSIDAD AUTÓNOMA DE
OCCIDENTE
COLOMBIA
enarvaez@uao.edu.co

Recibido: 27/XI/2015 / Aceptado: 31/III/2016

\section{Resumen}

Los estudios sobre lectura y escritura en educación superior cuentan con al menos dos décadas de desarrollo en Latinoamérica. Sin embargo, aún son escasas las investigaciones sistemáticas sobre la configuración de este espacio disciplinar. Con el propósito de llenar este vacío, el presente artículo identifica continuidades y contrastes locales e históricos a partir de un conjunto de variables (autores, idiomas, focos, temas, orientaciones, niveles, áreas, géneros y tipos). Para ello, un equipo internacional analizó cualitativa y cuantitativamente 81 artículos sobre el tema aparecidos en los últimos 15 años en cuatro revistas de Argentina, Brasil, Chile y Colombia consideradas líderes por la propia comunidad experta. Los resultados muestran una mayoría de artículos 
interesados en la investigación empírica de la escritura, en la formación de grado y el área de las Humanidades y las Ciencias Sociales, con foco en el artículo de investigación, con autores internacionales pero publicados en español y portugués. Históricamente, desapareció la lectura como competencia aislada y crecieron los enfoques híbridos y con mayor claridad epistemológica. Los contrastes regionales indican que la lectura y escritura son mayormente consideradas procesos educativos situados en la revista colombiana y discursos especializados en la argentina y chilena, con la brasileña entre medio. Además, los corpora brasileño y colombiano favorecen dinámicas de intercambio local mientras que los de Argentina y Chile presentan más autores internacionales. Esta reflexión disciplinar sobre los estudios de la lectura y la escritura en Latinoamérica resulta de importancia científica, educativa y política para su visibilización, validación, institucionalización y expansión.

Palabras Clave: Alfabetización académica, didáctica de la escritura, universidad, discurso académico, ILEES.

\begin{abstract}
Higher education reading and writing studies have developed for at least two decades in Latin America. However, little systematic research reveals the emerging shape of this disciplinary space. To fill this gap, this article compares publications from different countries and moments based on the variables of authors, languages, focuses, topics, orientations, levels, areas, genres and types. An international team analyzed qualitatively and quantitatively 81 articles on reading and writing published during the last 15 years in four leading journals according to the expert community from Argentina, Brazil, Chile and Colombia. Results show that most articles do empirical research on writing; they tend to study the development of undergraduate writing and prefer the Humanities and Social Sciences; they mostly focus on the research article; they are written by authors from various countries, in Spanish and Portuguese. From a historical point of view, reading as an isolated competence gradually disappeared and hybrid, epistemologically more explicit frameworks increased. Regional contrasts indicate that reading and writing are mostly understood as educationally situated processes in the Colombian journal and as specialized discourses in Argentinean and Chilean, with the Brazilian journal positioned in between. Furthermore, Brazilian and Colombian journals promote local researchers whereas Argentinean and Chilean offer more international authors. This disciplinary consideration on reading and writing studies in Latin America has scientific, educational and political relevance to foster disciplinary advance, validation, institutionalization and expansion.
\end{abstract}

Key Words: Academic literacy, teaching writing, university, academic discourse, ILEES.

\title{
INTRODUCCIÓN ${ }^{1}$
}

En las últimas décadas, se ha producido en Latinoamérica un conjunto de hitos institucionales, pedagógicos y científicos que permiten demostrar el desarrollo e impacto de lo que llamaremos el espacio disciplinar de los estudios sobre lectura y escritura en educación superior. Este espacio disciplinar responde a denominaciones diversas (como 'alfabetización académica', 'análisis del discurso académico' o 'didáctica 
de la escritura en educación superior') y se nutre de tradiciones teórico-metodológicas múltiples, con especial incidencia de los estudios del discurso, la lingüística aplicada, la psicología, las ciencias de la educación y las didácticas específicas. Con sus primeros antecedentes en los años 80, las iniciativas de enseñanza, los congresos, las investigaciones y publicaciones sobre la escritura en la educación superior se han multiplicado hasta constituir un campo diverso y en expansión.

En este contexto, resulta imprescindible llevar a cabo investigaciones que colaboren con la delimitación del espacio disciplinar de los estudios sobre lectura y escritura académicas en Latinoamérica a partir del análisis de sus canales de socialización, de temáticas y orientaciones teóricas, de los tipos de proyectos realizados, de las dinámicas de intercambio regionales y de los géneros y áreas disciplinares priorizados, entre otras prácticas relevantes. Los textos de investigación sobre lectura y escritura en educación superior, aparecidos en canales institucionales de intercambio científico de la región, constituyen un rico corpus para lograr este propósito. Existen escasas investigaciones que sigan este enfoque en la región (por ejemplo, Ortiz Casallas, 2011; Tapia-Ladino, 2013; Motta-Roth, 2014), si bien en otros entornos son más frecuentes y han tenido una fuerte influencia en el desarrollo de la disciplina (por ejemplo, Haswell, 2005; Mueller, 2012). En esa misma línea, los estudios históricos de la propia disciplina han sido frecuentes en los estudios de la composición norteamericana (Russell, 2006; Hawk, 2007; Miller, 2010; Gold, 2012). Tal como afirma Goggin (2000: xiv; traducción nuestra): "las historias disciplinares en particular juegan un rol crucial para legitimar las comunidades intelectuales y para ayudarlas a asegurarse un lugar en la academia".

Con el propósito de llenar este vacío, se conformó un equipo de siete investigadores de cinco países que relevaron y analizaron 81 artículos pertenecientes al área aparecidos en los últimos 15 años en las cuatro principales revistas científicas de Latinoamérica dedicadas a la temática según una encuesta voluntaria a miembros de la comunidad. Las preguntas de investigación del proyecto fueron las siguientes:

1. ¿Cómo se caracterizan las investigaciones sobre lectura y escritura en educación superior publicadas en América Latina durante los últimos 15 años?

2. ¿Cuáles son las continuidades y contrastes geográficos e históricos?

Para responder estas preguntas, se propuso un conjunto de variables emergentes de codificación cualitativa (autores, idiomas, focos, temas, orientaciones, niveles, áreas, géneros y tipos) y se cuantificó su distribución a lo largo de los corpora nacionales. Los resultados de esta investigación, basados en datos empíricos, describen los rasgos salientes de este espacio disciplinar y muestran contrastes entre los corpora según dinámicas geográficas e históricas. Así, este artículo hace aportes para comprender la historia y la situación actual de los estudios sobre lectura y escritura en educación superior en América Latina y para discutir sus direcciones futuras de desarrollo. 


\section{Contextualización histórica}

Un primer antecedente del interés por la temática puede situarse en 1980, cuando comenzó a publicarse en Argentina Lectura y Vida - Revista Latinoamericana de Lectura, la única publicación en español de la Asociación Internacional de Lectura; con foco en la lectura y en niveles educativos iniciales y medios, tuvo un gran impacto en la región hasta su desaparición en el año 2010 con 31 números publicados². Poco más tarde, en 1987, la cátedra de Semiología de la Universidad de Buenos Aires (Argentina), a cargo de Elvira Arnoux, llevó a cabo un proyecto pionero de investigación-acción para la enseñanza de la lectura y la escritura en la educación superior e institucionalizó, a partir de 2001, los influyentes Talleres de Lectura y Escritura del Ciclo Básico Común (Pereira, 2006).

En el marco de la expansión del acceso a la educación superior en la región de las últimas décadas y los desafíos que las nuevas poblaciones estudiantiles representan (UNESCO, 2009; Ezcurra, 2011; Fernández Lamarra \& Costa de Paula, 2011), distintas universidades latinoamericanas pusieron en marcha cursos curriculares y extracurriculares de enseñanza de la lectura y la escritura académicas en diferentes etapas de la educación superior desde los años 90 y, en particular, desde mediados de los años 2000 (ver, por ejemplo, Narváez Cardona, 2012; Navarro, 2012; Ávila Reyes, González-Álvarez \& Peñaloza Castillo, 2013). Este proceso multiplicó la comunidad de docentes interesados y especializados en la temática y, al ser un espacio de docencia e investigación novedoso, favoreció los vínculos e intercambios interdisciplinarios.

Entre 1994 y 1996, se creó la Cátedra UNESCO para el Mejoramiento de la Calidad y Equidad de la Educación en América Latina con base en la Lectura y Escritura a partir de un acuerdo entre la Universidad del Valle (Colombia), su sede principal, el Instituto Caro y Cuervo de Santafé de Bogotá (Colombia), la Universidad de Buenos Aires (Argentina) y la Pontificia Universidad Católica de Valparaíso (Chile). Esta red se ha ampliado desde entonces también a México, Perú, Venezuela, Puerto Rico, Costa Rica, Ecuador y Brasil, con un total de 44 sedes en 10 países. La misma Cátedra UNESCO realizó en 1998 el Primer Simposio en Buenos Aires y en 2001 el Primer Congreso Internacional organizado por la Universidad del Valle (Ortega de Hocevar, 2015). Desde sus inicios, una importante línea temática de sus encuentros ha sido el espacio de la educación superior. Paralelamente, este interés se masificó en líneas temáticas, mesas, conferencias y simposios dentro de otros congresos de la región. La evidente importancia de la Cátedra UNESCO en Latinoamérica permite señalar un punto de inicio claro de este espacio disciplinar a mediados de los años 90.

En 2003 se creó en la Universidade Estadual de Londrina (Brasil) el Simpósio Internacional de Estudos de Gêneros Textuais (SIGET), un evento internacional que busca discutir el rol de los géneros discursivos y/o textuales en las prácticas sociales y que alcanzó en 2015 su octava edición ${ }^{3}$, con espacios temáticos dedicados al estudio de la 
escritura profesional y universitaria. En 2006, se creó en Colombia la Red de Lectura y Escritura en Educación Superior (REDLESS), que en 2007 celebró su Primer Encuentro Nacional sobre Políticas Institucionales para el Desarrollo de la Lectura y Escritura en la Educación Superior, el cual se ha venido repitiendo desde entonces ${ }^{4}$.

Diversas publicaciones acompañaron estos procesos de expansión y visibilidad del interés por la lectura y la escritura en educación superior. A comienzos de los años 2000, se publicaron algunos artículos que adoptaban una primera mirada reflexiva sobre estas iniciativas institucionales, pedagógicas y científicas (Carlino, 2003). También aparecieron volúmenes colectivos sobre la lectura y la escritura en educación superior como objeto de investigación y enseñanza, tanto en formato de libros (Parodi, 2010) o volúmenes monográficos (Cubo de Severino, 2005; Carrasco, Encinas, Castro \& López Bonilla, 2013), además de profusos materiales didácticos (Machado, Lousada \& Tardelli, 2005; Venegas, Núñez, Zamora \& Santana, 2015). Las temáticas también se fueron especializando en las revistas del área, de modo que ya en la década del 2000 se podían encontrar múltiples trabajos sobre lectura y escritura terciarias. El caso de Lectura y Vida, señalada como pionera, es paradigmático: mientras el primer número de 1980 mostraba un marcado énfasis en temáticas como la ortografía y la lectura en el nivel escolar primario y con una perspectiva cognitivoexperimental, su último número, de 2010, demuestra que en tres décadas los intereses se desplazaron a los niveles educativos medio y superior y al análisis de géneros discursivos, iniciativas y representaciones desde una perspectiva preferentemente sociocultural.

De forma más reciente, en 2014 se publicó el primer número de Traslaciones. Revista Latinoamericana de Lectura y Escritura, publicación científica oficial de la Cátedra UNESCO. En el mismo año, en Colombia se fundó la Red Latinoamericana de Centros y Programas de Escritura, que ha realizado hasta la fecha dos congresos latinoamericanos ${ }^{5}$, y se creó en la Universidad Tecnológica de Pereira la Base internacional bibliográfica sobre lectura y escritura, de construcción colaborativa y disponible en línea ${ }^{6}$. Estos hitos institucionales se completan con la aparición en la región de numerosos programas de posgrado dedicados a la temática.

Además, en los últimos años se han conformado equipos y proyectos de investigación que comenzaron a reflexionar sistemática y contrastivamente sobre el propio desarrollo de este espacio disciplinar atendiendo a las iniciativas de enseñanza de la lectura y la escritura en la región, como el grupo de investigación internacional Iniciativas de Lectura y Escritura en la Educación Superior en Latinoamérica (ILEES; Bazerman, Ávila-Reyes, Bork, Corrêa, Cristovão, Tapia-Ladino \& Narváez, en prensa). En esta misma línea de desarrollo y proyección regional e internacional, en 2017 se celebrará en Colombia el IV Writing Research Across Borders (WRAB), uno de los eventos más importantes del mundo en el área. Diversas experiencias de escritura de la región han quedado plasmadas en libros recopilatorios internacionales, tales como 
Bazerman et al. (en prensa) y Thaiss, Bräuer, Carlino, Ganobcsik-Williams \& Sinha (2012).

En suma, el contraste histórico muestra un aumento progresivo de iniciativas de enseñanza, investigación, publicación, eventos y esfuerzos de sistematización, que hacen necesario un recuento basado en datos que contribuya a conocer mejor y consolidar el espacio disciplinar de la lectura y la escritura universitarias.

\section{Marco teórico}

Para conceptualizar la ciencia como un ámbito social con condiciones específicas de producción, Bourdieu (2003) hace una analogía con el mercado y plantea que en los 'campos científicos' existe una competencia entre 'productores' (los científicos) y sus 'productos' (las verdades científicas) por conseguir un 'capital' (el monopolio de la autoridad científica). Los intercambios científicos se conceptualizan como pugnas para conseguir prestigio, autoridad y legitimidad y así lograr una mayor capacidad de intervenir frente a los competidores (los pares expertos) en materia de ciencia (ver también Becher, 2001). Este capital que poseen los científicos puede ser no solo académico, sino también político y burocrático. Se entiende entonces que la configuración de los campos científicos depende de la relación de fuerzas entre los capitales de los científicos en un momento determinado. Esta concepción del campo científico como espacio de disputa de intereses enfrentados encuentra su balance en la doxa: el "conjunto de presupuestos que los antagonistas admiten de hecho, sin discusión" (Bourdieu, 2003: 44). Así, la doxa identifica los acuerdos y consensos implícitos del campo en un marco de disputa y cambio.

Bajo esta perspectiva general sobre las dinámicas de los campos científicos, resulta consistente conceptualizar las disciplinas como formaciones sociales con base institucional y curricular en carreras, departamentos, revistas y congresos, entre otros, que garantizan la legitimización, el control y la reproducción de la disciplina (Goggin, 2000). Según Jacobs (2013; traducción nuestra):

"una disciplina es una forma de organización social que genera nuevas ideas y hallazgos de investigación, certifica ese conocimiento y a su vez enseña esos contenidos a los estudiantes interesados".

A su vez, las disciplinas poseen prácticas, formas de comunicación, conocimientos consensuados y acuerdos sobre la forma de disputar esos acuerdos y resolver los disensos (Hyland, 2011).

Pero esta visión sobre las continuidades y elementos comunes de las disciplinas debe complementarse con una atención por los procesos de mutación, hibridación, variación regional e interdisciplinariedad característicos de las dinámicas del intercambio científico. Según Klein (1996), la hibridación se produce cuando las 
comunidades interactúan en zonas disciplinarmente limítrofes. Allí, pueden desarrollar una 'lengua pidgin' para el intercambio y negociación esporádica entre disciplinas, o una verdadera 'lengua creole' como patrimonio discursivo de su nueva identidad profesional. Algunos de los promotores de la permeabilidad entre fronteras disciplinares son los préstamos de métodos de análisis y conceptos teóricos, la identificación de problemas de investigación fuera del recorte disciplinar estricto y la redefinición de qué es lo intrínseco de cada disciplina.

Abordar las disciplinas desde un marco teórico que identifique los consensos y prácticas compartidas y las bases institucionales de apoyo (en particular, las revistas científicas más influyentes), pero que al mismo tiempo dé cuenta de las pugnas por el capital simbólico y los procesos de hibridación teórica, es apropiado para el caso de los estudios sobre lectura y escritura en educación superior en América Latina. En efecto, este espacio disciplinar, de desarrollo relativamente reciente, muestra avances exponenciales en la cantidad de conocimiento consensuado acumulado y en las bases institucionales que lo sostienen, pero se caracteriza por objetos de estudio que atraviesan áreas y que generan interés en investigadores de diferentes disciplinas.

\section{Metodología}

Los autores de este artículo se organizaron en diferentes roles: un director de proyecto de investigación general, un investigador principal, una investigadora metodóloga y cuatro equipos para la construcción y análisis de los corpora por países.

\subsection{Procedimientos de obtención del corpus}

El primer paso para la construcción del corpus consistió en la determinación de las revistas científicas de las que se extraerían los artículos de investigación. Dado que no existen sistemas de evaluación consensuados en la región para determinar contrastivamente y en detalle la calidad de las publicaciones periódicas, se utilizaron las encuestas realizadas por el proyecto de investigación ILEES (Bazerman et al., en prensa; Bork, Bazerman, Correa \& Cristovão, 2014). Estas fueron realizadas entre junio de 2012 y septiembre de 2013 a 118 académicos de 7 países, mediante muestreo intencionado y técnica de 'bola de nieve'. Se incluyeron dos preguntas tendientes a identificar los canales de socialización científica, una sobre recursos utilizados y otra sobre las revistas más apropiadas para divulgar el trabajo del área. La primera de estas preguntas arrojó un alto grado de dispersión, además de revelar una escasa mención a bases de datos académicas y una importante recurrencia de fuentes no institucionales, tales como blogs y sitios web. En cuanto a la segunda pregunta, el 50\% de 243 menciones no consiguió identificar una revista específica para el tema. De entre las menciones válidas, $45 \%$ se trató de menciones únicas y las restantes se distribuyeron entre 31 títulos. Ambas respuestas mostraron un desarrollo incipiente de las prácticas de publicación en este espacio disciplinar y la falta de una publicación periódica específica y reconocida. 
Con los resultados válidos de la segunda pregunta, se identificó como las revistas más apropiadas para divulgar el trabajo del campo, en orden alfabético de países, Signo y Seña (Universidad de Buenos Aires, Argentina) con 7 menciones únicas; Revista Brasileira de Linguistica Aplicada (Universidade Federal de Minais Gerais, Brasil) con 9 menciones únicas; Revista Signos. Estudios de Lingüistica (Pontificia Universidad Católica de Valparaíso, Chile) con 24 menciones únicas; y Lenguaje (Universidad del Valle, Colombia) con 9 menciones.

La revista Signo y Seña (SyS) es una publicación científica periódica con referato del Instituto de Lingüística de la Universidad de Buenos Aires dedicada a "investigaciones de todas las áreas de la lingüística"7 e indexada en Núcleo Básico de Revistas Científicas Argentinas y Latindex, entre otras. Publica artículos, notas y reseñas en español y portugués. Entre 2001 y 2009 publicó en papel 10 números monográficos, con especialistas como coordinadores, y desde 2012 ha cambiado a formato electrónico de libre acceso y números semestrales con una sección temática (dossier) a cargo de especialistas del área. La mayor parte del corpus argentino proviene de dos números monográficos publicados en 2005 y 2006.

La Revista Brasileira de Lingüistica Aplicada (RBLA) es una publicación científica periódica trimestral con referato y acceso libre en línea de la Universidade Federal de Minais Gerais que "tiene la misión de fomentar la investigación en el área de

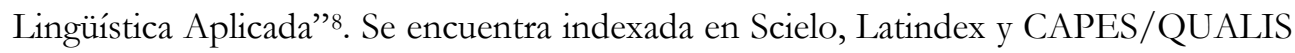
(A1), entre otras. Publica artículos, reseñas, ensayos, entrevistas y dos números temáticos al año. Se lanzó en 2001 como iniciativa de la Associação de Linguística Aplicada do Brasil con un único volumen y luego se publicó semestralmente entre 2002 y 2009 y trimestralmente desde 2010.

Revista Signos. Estudios de Lingüistica es una publicación científica periódica cuatrimestral con referato y acceso libre en línea desde 2012 de la Pontificia Universidad Católica de Valparaíso que publica artículos y reseñas en español e inglés. Dedicada desde 1967 a las ciencias del lenguaje y la literatura, en 2005 "acota su enfoque disciplinar exclusivamente al área de la lingüística y a algunas interdisciplinas: Psicolingüística, Lingüística del Texto y del Discurso y Lingüística Aplicada"9. Se encuentra indexada en ISI Web of Knowledge, Scielo y Scopus, entre otras.

La revista Lenguaje, de la Escuela de Ciencias del Lenguaje de la Universidad del Valle, es una publicación científica periódica semestral con referato indexada en Publíndex de Colciencias (B) y Latindex, entre otras. Fue creada en 1972 y publica artículos y reseñas "en cualquiera de las disciplinas que se ocupan del lenguaje"10 de forma anual hasta 2005 y de forma semestral desde 2006.

En suma, estas revistas pertenecen institucionalmente a universidades de prestigio en sus respectivos países y que, en el caso de Argentina, Chile y Colombia, constituyen 
los centros nacionales fundacionales de la Cátedra UNESCO. Sin embargo, más allá de la importancia institucional y científica de las revistas y países seleccionados, es probable que algunos de los resultados no representen tendencias a nivel nacional y regional sino preferencias idiosincráticas de las revistas o instituciones. Por otro lado, las afirmaciones sobre el desarrollo de este espacio disciplinar en la región deberían incluir otros países que no fueron considerados en nuestro recorte metodológico. A pesar de estas limitaciones, son numerosas las investigaciones que seleccionan investigadores, canales o centros de investigación de relevancia histórica como estrategia metodológica para obtener indicios bien fundamentados y abordables que ayuden a comprender las características y desarrollo de culturas disciplinares y prácticas letradas más amplias (ver, por ejemplo, Halliday, 2004). Así, algunos de los hallazgos de este trabajo pueden servir para comprender los estudios sobre lectura y escritura en educación superior en Latinoamérica.

El período de tiempo considerado es entre 2001 (fecha de publicación del primer número de la revista brasileña) y el primer semestre de 2015: 15 años. El inicio de este período coincide parcialmente con algunos hitos institucionales de desarrollo de los estudios de la lectura y la escritura en la región mencionados previamente.

A partir de las revistas seleccionadas, los equipos autorales organizados por países cuantificaron el total de artículos científicos completos y seleccionaron y digitalizaron aquellos artículos dedicados a la lectura y la escritura en primera lengua (independientemente de cuál sea esta) en educación superior; no se consideraron reseñas, notas ni artículos breves. El foco no está en investigaciones sobre fenómenos latinoamericanos, sino en investigaciones publicadas en la región que pueden tomar como objeto fenómenos locales o internacionales. Para delimitar con claridad el alcance del corpus, los artículos seleccionados debieron cumplir con los siguientes criterios, basados parcialmente en Ávila Reyes (2015):

- Toman como objeto de estudio la lectura y la escritura avanzadas (académicas, universitarias, científicas o profesionales) como proceso (por ejemplo, la enseñanza de la lectura en el aula de biología) o como producto (por ejemplo, la presencia de metáforas en artículos de investigación).

- Si abordan la lectura y la escritura en general o de otros niveles educativos, también consideran las prácticas de las esferas académicas y profesionales.

- Estudian la lectura y la escritura en L1 o el contraste entre L1 y L2, si bien pueden estar escritos en cualquier lengua. Los artículos que solo abordan la lectura y la escritura en L2 no son considerados.

Luego de la construcción de los corpora siguiendo estos criterios, los investigadores principales revisaron la selección para descartar la aparición de falsos negativos o positivos. Posteriormente a este proceso, se realizaron los ajustes 
necesarios hasta concluir en los corpora revisados, los cuales se componen de la siguiente forma:

Tabla 1. Cantidad relativa y absoluta de artículos sobre lectura y escritura en educación superior en cada corpus.

\begin{tabular}{|l|c|c|c|}
\hline & $\mathbf{N}^{\mathbf{o}}$ artículos corpus & $\mathbf{N}^{\mathbf{0}}$ artículos revista & \% corpus/revista \\
\hline SyS (Argentina) & 16 & 177 & 9,0 \\
\hline RBLA (Brasil) & 15 & 396 & 3,8 \\
\hline Signos (Chile) & 23 & 270 & 8,5 \\
\hline Lenguaje (Colombia) & 27 & 215 & 12,6 \\
\hline TOTAL & $\mathbf{8 1}$ & $\mathbf{1 0 5 8}$ & $\mathbf{7 , 7}$ \\
\hline
\end{tabular}

El corpus total incluye 81 artículos científicos, que representan el 7,7\% del total de artículos aparecidos en las cuatro revistas consideradas.

\subsection{Procedimientos de análisis}

El análisis cualitativo se realizó mediante la técnica del análisis de contenido. Cada equipo autoral por países realizó un análisis cualitativo a partir de un conjunto de categorías fijas basadas en un análisis exploratorio del corpus y de algunas hipótesis deductivas previas. Posteriormente, los investigadores principales descartaron algunas categorías cualitativas que resultaron poco productivas o difíciles de comparar (por ejemplo, 'metodología' y 'principales hallazgos') y desarrollaron un conjunto de valores de codificación emergentes para cuantificar categorías cualitativas (Glässer \& Laudel, 2013). Así, por ejemplo, a la categoría abierta Tema, que en cada uno de los 81 artículos analizados arrojó diversas descripciones cualitativas de contenidos, se le atribuyeron seis valores emergentes posibles (ver Tabla 2). Los códigos se validaron en tres etapas: primero fueron propuestos por la analista metodóloga a partir del análisis de los equipos por países, luego fueron validados por el investigador principal y por último fueron convalidados por los autores por países que habían realizado los análisis originales, quienes sugirieron modificaciones o ajustes a la codificación. Los valores obtenidos fueron cuantificados mediante el software IBM Statistical Package for Social Sciences, utilizando conteos de frecuencia y tablas de contingencia. La Tabla 2 muestra las categorías de análisis y los códigos considerados: 
Tabla 2. Categorías y valores de análisis cualitativo y cuantitativo de contenidos de los corpora.

\begin{tabular}{|c|c|c|c|}
\hline $\mathbf{N}^{\mathbf{o}}$ & Categoría & Descripción & Codificación \\
\hline 1 & Año & $\begin{array}{l}\text { Año de publicación } \\
\text { del artículo. }\end{array}$ & $2001-2015$ \\
\hline \multirow[t]{4}{*}{2} & \multirow[t]{4}{*}{ País artículo } & \multirow{4}{*}{$\begin{array}{l}\text { País de publicación } \\
\text { del artículo. }\end{array}$} & Argentina \\
\hline & & & Brasil \\
\hline & & & Chile \\
\hline & & & Colombia \\
\hline \multirow[t]{7}{*}{3} & \multirow{7}{*}{$\begin{array}{l}\text { Origen } \\
\text { institucional } \\
\text { autor }\end{array}$} & \multirow{7}{*}{$\begin{array}{l}\text { Filiación } \\
\text { institucional de los } \\
\text { autores (tal como } \\
\text { aparece en el } \\
\text { artículo) } \\
\text { independientemente } \\
\text { de su nacionalidad. }\end{array}$} & Argentina \\
\hline & & & Brasil \\
\hline & & & Chile \\
\hline & & & Colombia \\
\hline & & & $\begin{array}{l}\text { Otro LA: autor o autores de países } \\
\text { latinoamericanos que no son Argentina, Brasil, } \\
\text { Chile ni Colombia. }\end{array}$ \\
\hline & & & $\begin{array}{l}\text { Otro extranjero: autor o autores de países no } \\
\text { latinoamericanos. }\end{array}$ \\
\hline & & & $\begin{array}{l}\text { Mixto: autores de diversos países que combinan } \\
\text { las categorías previas. }\end{array}$ \\
\hline \multirow[t]{4}{*}{4} & \multirow[t]{4}{*}{ País/origen } & \multirow{4}{*}{$\begin{array}{l}\text { Grado de } \\
\text { endogamia o } \\
\text { exogamia a partir del } \\
\text { análisis de la } \\
\text { coincidencia o } \\
\text { divergencia entre } \\
\text { país del artículo y } \\
\text { origen del autor o } \\
\text { autores, } \\
\text { respectivamente. }\end{array}$} & $\begin{array}{l}\text { Mismo: coincide el país del artículo con el } \\
\text { origen institucional del autor o autores. }\end{array}$ \\
\hline & & & $\begin{array}{l}\text { Otro LA: el autor o autores tienen filiación } \\
\text { latinoamericana en un país distinto del artículo. }\end{array}$ \\
\hline & & & $\begin{array}{l}\text { Otro extranjero: el autor o autores tienen } \\
\text { filiación no latinoamericana. }\end{array}$ \\
\hline & & & $\begin{array}{l}\text { Múltiple: autores de diversos países que } \\
\text { combinan las categorías previas (por ejemplo, } \\
\text { un autor coincide con el país del artículo pero } \\
\text { otro es no latinoamericano). }\end{array}$ \\
\hline \multirow[t]{3}{*}{5} & \multirow[t]{3}{*}{ Idioma } & \multirow{3}{*}{$\begin{array}{l}\text { Idioma en que se } \\
\text { publica el artículo } \\
\text { sin importar el } \\
\text { idioma en que se } \\
\text { escribió } \\
\text { originalmente. }\end{array}$} & Español \\
\hline & & & Portugués \\
\hline & & & Inglés \\
\hline \multirow[t]{2}{*}{6} & \multirow[t]{2}{*}{$\begin{array}{l}\text { Idioma/ } \\
\text { origen }\end{array}$} & \multirow{2}{*}{$\begin{array}{l}\text { Relación entre el } \\
\text { idioma del artículo y } \\
\text { el idioma que se } \\
\text { habla en el país de } \\
\text { origen institucional } \\
\text { del autor. }\end{array}$} & $\begin{array}{l}\text { Mismo: coincide el idioma del artículo con el } \\
\text { idioma que se habla en el país de origen } \\
\text { institucional del autor o autores. }\end{array}$ \\
\hline & & & $\begin{array}{l}\text { Distinto: no coincide el idioma del artículo con } \\
\text { el idioma que se habla en el país de origen } \\
\text { institucional del autor o autores. }\end{array}$ \\
\hline \multirow[t]{3}{*}{7} & \multirow[t]{3}{*}{ Foco } & \multirow{3}{*}{$\begin{array}{l}\text { Foco en lectura, en } \\
\text { escritura o en } \\
\text { ambas. }\end{array}$} & Lectura \\
\hline & & & Escritura \\
\hline & & & Lectura y escritura \\
\hline \multirow[t]{4}{*}{8} & \multirow[t]{4}{*}{ Tema } & \multirow[t]{4}{*}{$\begin{array}{l}\text { Tema o problema de } \\
\text { investigación. }\end{array}$} & $\begin{array}{l}\text { Lec/esc ens/inv: fundamentación teórica o } \\
\text { empírica para validar la lectura o la escritura en } \\
\text { educación superior como objeto de estudio y/o } \\
\text { de enseñanza. }\end{array}$ \\
\hline & & & $\begin{array}{l}\text { Géneros: caracterización de uno o más géneros } \\
\text { discursivos y/o textuales. }\end{array}$ \\
\hline & & & $\begin{array}{l}\text { Discurso: fenómenos discursivos o textuales } \\
\text { tales como la modalidad, la evaluación o la } \\
\text { intertextualidad. }\end{array}$ \\
\hline & & & Enseñanza: análisis sobre métodos y estrategias \\
\hline
\end{tabular}




\begin{tabular}{|c|c|c|c|}
\hline & & & $\begin{array}{l}\text { para enseñar la lectura o la escritura en } \\
\text { educación superior. }\end{array}$ \\
\hline & & & $\begin{array}{l}\text { Ambientes virtuales: lectura y escritura en } \\
\text { soportes digitales o a través del uso de TIC para } \\
\text { el aprendizaje. }\end{array}$ \\
\hline & & & $\begin{array}{l}\text { Lec/esc alumnos: análisis del desempeño } \\
\text { escrito o lector de los estudiantes o de sus } \\
\text { textos como productos. }\end{array}$ \\
\hline 9 & Orientación & Disciplinas, & Etnografía (e.g., Ruth Sautu) \\
\hline & teórica & $\begin{array}{l}\text { tradiciones y marcos } \\
\text { teórico- }\end{array}$ & $\begin{array}{l}\text { NEL: Nuevos Estudios de Literacidad (e.g., } \\
\text { James Gee). }\end{array}$ \\
\hline & & metodológicos. & $\begin{array}{l}\text { LSF: Lingüística Sistémico-Funcional (e.g., } \\
\text { Michael Halliday). }\end{array}$ \\
\hline & & & $\begin{array}{l}\text { ISD: Interaccionismo Socio-Discursivo (e.g., } \\
\text { Jean Paul Bronckart). }\end{array}$ \\
\hline & & & $\begin{array}{l}\text { LFE: Lenguas para Fines Específicos: incluye } \\
\text { inglés para fines específicos (English for Specific } \\
\text { Purposes) (e.g., John Swales). }\end{array}$ \\
\hline & & & Multimodalidad (e.g., Gunther Kress). \\
\hline & & & $\begin{array}{l}\text { ERG: Estudios Retóricos del Género (Rhetorical } \\
\text { Genre Analysis) (e.g., Caroline Miller). }\end{array}$ \\
\hline & & & $\begin{array}{l}\text { Corpus: Lingüística de Corpus (e.g., Douglas } \\
\text { Biber). }\end{array}$ \\
\hline & & & $\begin{array}{l}\text { Ling. Textual: Lingüística Textual en sentido } \\
\text { amplio; incluye gramática, teoría de la } \\
\text { enunciación, estudios del discurso y lingüística } \\
\text { textual de tradición alemana (e.g., Catherine } \\
\text { Kerbrat-Orecchioni). }\end{array}$ \\
\hline & & & $\begin{array}{l}\text { Cognitivismo: Teorías Cognitivas en sentido } \\
\text { amplio; incluye modelos de procesamiento de la } \\
\text { lectura y la escritura y estudios de teorías } \\
\text { implícitas (e.g., Walter Kintsch). }\end{array}$ \\
\hline & & & $\begin{array}{l}\text { Esc. Disciplinas: Escritura en las Disciplinas; } \\
\text { incluye movimientos escribir a través del } \\
\text { currículum (Writing across the Curriculum) y } \\
\text { escribir en las disciplinas (Writing in the } \\
\text { Disciplines) (e.g., Paula Carlino). }\end{array}$ \\
\hline & & & $\begin{array}{l}\text { Mixta: combinación explícita de dos o más de } \\
\text { las orientaciones previas. }\end{array}$ \\
\hline & & & $\begin{array}{l}\text { N/D: no declarada; no aparece una orientación } \\
\text { que resulte evidente a partir de las referencias } \\
\text { bibliográficas ni una enunciación explícita en el } \\
\text { texto. }\end{array}$ \\
\hline 10 & Nivel & Nivel o esfera & Grado \\
\hline & & educativa y de uso. & Postgrado \\
\hline & & & Discurso experto \\
\hline & & & Multinivel \\
\hline & & & N/A: no aplica. \\
\hline 11 & Área & Área disciplinar & Profesores: cursos de formación docente. \\
\hline & & $\begin{array}{l}\text { investigada por el } \\
\text { artículo. }\end{array}$ & $\begin{array}{l}\text { Lenguas: cursos de enseñanza de lenguas } \\
\text { segundas y extranjeras. }\end{array}$ \\
\hline & & & $\begin{array}{l}\text { Letras: incluye estudios literarios y ciencias del } \\
\text { lenguaje. }\end{array}$ \\
\hline & & & Ingeniería \\
\hline & & & Humanidades \\
\hline & & & Educación \\
\hline
\end{tabular}




\begin{tabular}{|c|c|c|c|}
\hline & & & Economía \\
\hline & & & Psicología \\
\hline & & & Salud: ciencias de la salud. \\
\hline & & & Ciencias: incluye ciencias exactas y naturales. \\
\hline & & & Múltiples: más de un área disciplinar. \\
\hline & & & N/A: no aplica. \\
\hline & & & N/D: no declarada. \\
\hline 12 & Género & $\begin{array}{l}\text { Género discursivo } \\
\text { y/o textual } \\
\text { investigado por el }\end{array}$ & $\begin{array}{l}\text { Reseña: reseñas de diversos tipos, incluyendo } \\
\text { académicas y periodísticas, como género de } \\
\text { formación en educación superior. }\end{array}$ \\
\hline & & artículo. & AI: artículo de investigación científica. \\
\hline & & & Autobiografía: elaborada por estudiantes. \\
\hline & & & $\begin{array}{l}\text { Informe: informes de distintos tipos, incluyendo } \\
\text { informes bibliográficos, curriculares o } \\
\text { científicos, como género de formación en } \\
\text { educación superior. }\end{array}$ \\
\hline & & & Resumen: elaborado por estudiantes. \\
\hline & & & Foro: foro virtual en el que escriben estudiantes. \\
\hline & & & $\begin{array}{l}\text { Tesis: tesis de licenciatura, maestría y } \\
\text { doctorado; incluye proyectos de tesis. }\end{array}$ \\
\hline & & & Manual: leído por estudiantes. \\
\hline & & & $\begin{array}{l}\text { Otros: géneros no considerados en las } \\
\text { categorías previas que aparecen una sola vez en } \\
\text { el corpus. }\end{array}$ \\
\hline & & & Múltiples: más de un género. \\
\hline & & & $\begin{array}{l}\text { N/A: no aplica; cuando el género leído o escrito } \\
\text { no es relevante para la investigación o cuando } \\
\text { no hay ningún género determinado. }\end{array}$ \\
\hline & & & $\begin{array}{l}\text { N/D: cuando el género no aparece declarado o } \\
\text { especificado, por ejemplo cuando se trata de } \\
\text { "tareas escritas" o "textos estudiantiles". }\end{array}$ \\
\hline 13 & Tipo & Tipo de & Teórico: discusión y elaboración teórica. \\
\hline & & investigación & Empírico: investigaciones de base empírica. \\
\hline & & realizada. & $\begin{array}{l}\text { Aplicado: propuestas de intervención } \\
\text { pedagógica con aporte de datos y metodología } \\
\text { investigativa (por ejemplo, criterios de diseño de } \\
\text { cursos, pruebas a estudiantes antes y después de } \\
\text { un curso, etc.) }\end{array}$ \\
\hline & & & $\begin{array}{l}\text { Experiencial: relatos o reflexiones de } \\
\text { experiencias sin aporte de datos o con aporte } \\
\text { escaso de datos. }\end{array}$ \\
\hline
\end{tabular}

\section{Resultados y discusión}

El primer contraste a investigar es el peso relativo del corpus de artículos sobre lectura y escritura en educación superior en relación con la producción total de la revista de cada país. Según se ve en la Tabla 1, los resultados muestran que la incidencia del interés por la lectura y la escritura en educación superior en las revistas investigadas ronda el $8 \%$ y oscila entre el 3,8 y el $12,6 \%$, con mayor peso en el corpus colombiano y menor en el corpus brasileño. Al tratarse de revistas no dedicadas exclusivamente a la temática, esta incidencia relativamente baja no es sorprendente. La 
distribución de los corpora puede también analizarse desde un punto de vista histórico, como se ve en el Gráfico 1 a continuación:

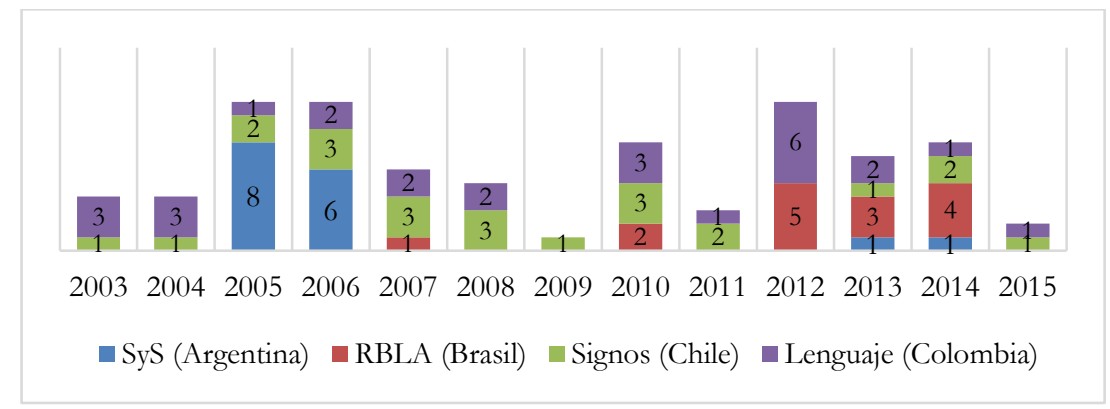

Gráfico 1. Cantidad de artículos sobre lectura y escritura en lengua primera en educación superior por revista y año.

Lo que muestra el Gráfico 1 es un interés constante pero móvil durante el período considerado: se ven máximos en los años 2005, 2006 y 2012, y marcados descensos en la cantidad en 2009, 2011 y 2015 (si bien este último año solo fue considerado durante el primer semestre); nótese además que no aparecen artículos en los dos primeros años considerados. Al distinguir entre países, las revistas colombiana y chilena muestran un interés permanente por la temática, mientras que las publicaciones de Argentina y Brasil se concentran en algunos años (Signo y Seña con dos números monográficos en 2005 y 2006; RBLA entre 2012 y 2014, con un interés reciente y creciente).

Para analizar las dinámicas de intercambio locales y regionales de los países considerados, es útil dar cuenta del grado relativo de endogamia y exogamia de los corpora, es decir, en qué medida los autores tienen un origen institucional en el mismo país de las revistas en las que publican:

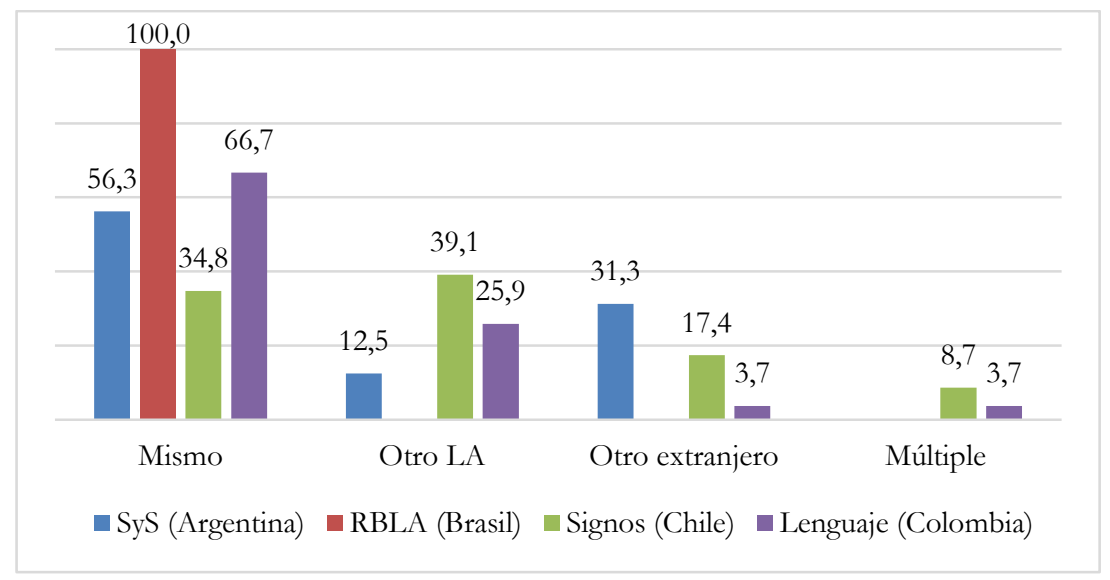

Gráfico 2. Relación entre país del artículo y origen institucional del autor en porcentaje. 
El Gráfico 2 muestra que las revistas chilena y, en menor medida, argentina son las más exogámicas, esto es, con mayor presencia de autores de otros países, si bien Revista Signos. Estudios de Lingḯstica muestra una distribución balanceada entre autores chilenos, latinoamericanos y no latinoamericanos, mientras que Signo y Seña prioriza autores argentinos y no latinoamericanos. La RBLA, por el contrario, es la publicación más endogámica, con un $100 \%$ de autores con origen institucional en Brasil, mientras que Lenguaje de Colombia no presenta autores no latinoamericanos. Cabe agregar que, salvo dos artículos en inglés (uno en Chile de investigadores estadounidenses y otro en Colombia de una investigadora mexicana), todos los artículos están escritos en el idioma del país en el que se publican, algunos de ellos como traducciones de otros idiomas.

Para ahondar en esta variable, se analizan y cuantifican los artículos en los que al menos uno de los autores pertenece a la misma institución que publica la revista:

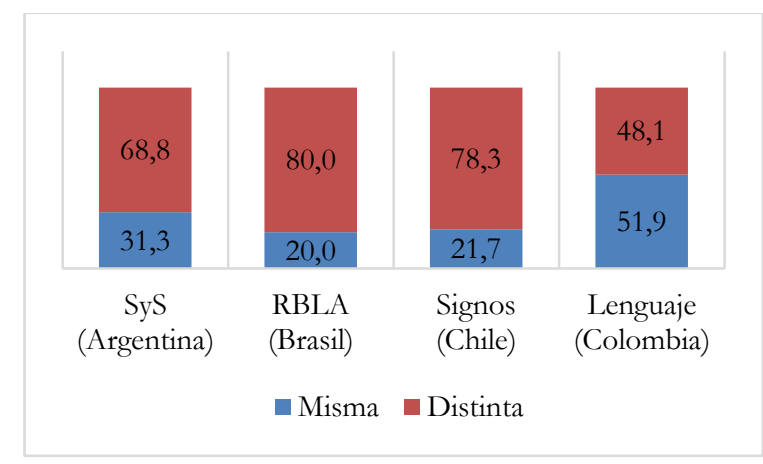

Gráfico 3. Relación entre institución de pertenencia de revista y autor en porcentaje.

El Gráfico 3 complejiza el panorama previo. Puede apreciarse que el corpus brasileño, a pesar de su endogamia nacional (Gráfico 2), muestra aquí la menor endogamia institucional de todos los corpora: el $80 \%$ de los autores no pertenecen a la misma institución que la revista en la que publican; este dato no es sorprendente ya que constituye un criterio explícito de evaluación de la calidad de las publicaciones científicas en el país. El corpus chileno, por su parte, se ubica muy cerca, con un $78,3 \%$ de los autores. En el otro extremo de la escala, algo más de la mitad de los autores que publican en el corpus colombiano pertenecen a la misma institución que la revista en la que publican; Argentina se coloca en una posición intermedia entre Colombia y el resto de los países, con casi uno de cada tres autores de la misma institución que la revista correspondiente.

Asumiendo un punto de vista histórico, el Gráfico siguiente permite identificar el peso relativo de la lectura, la escritura y la lectura y escritura durante los últimos 15 años: 


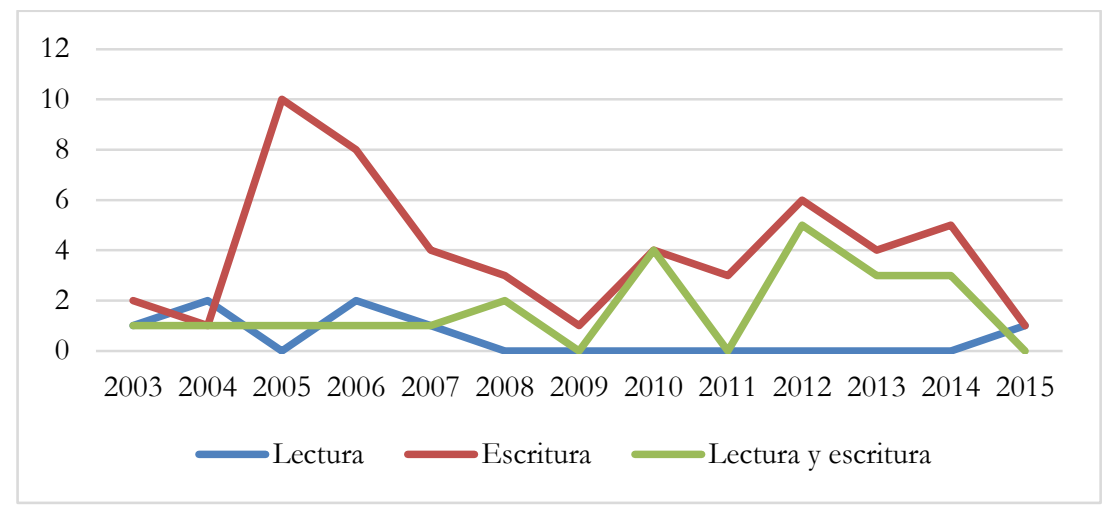

Gráfico 4. Cantidad de artículos por foco y año.

Como se aprecia, la mayoría de los artículos (n. 52; 64,2\%) se centran solo en la escritura, o bien como proceso o bien como producto en el discurso escrito. La lectura por sí misma, en cambio, no es un foco de investigación prioritario (n. 7; 8,6\%), a diferencia de lo que sucedía en las décadas previas en la región (cuando, por ejemplo, existía la revista Lectura y Vida). De hecho, en 2003 y 2004 hay una virtual coincidencia entre los tres focos analizados, mientras que, a partir de 2005, estos se diferencian con mayor claridad.

Los artículos del corpus se subclasificaron en seis temas de investigación para poder determinar los objetos de indagación priorizados regional y localmente. El siguiente Gráfico permite verificar los resultados:

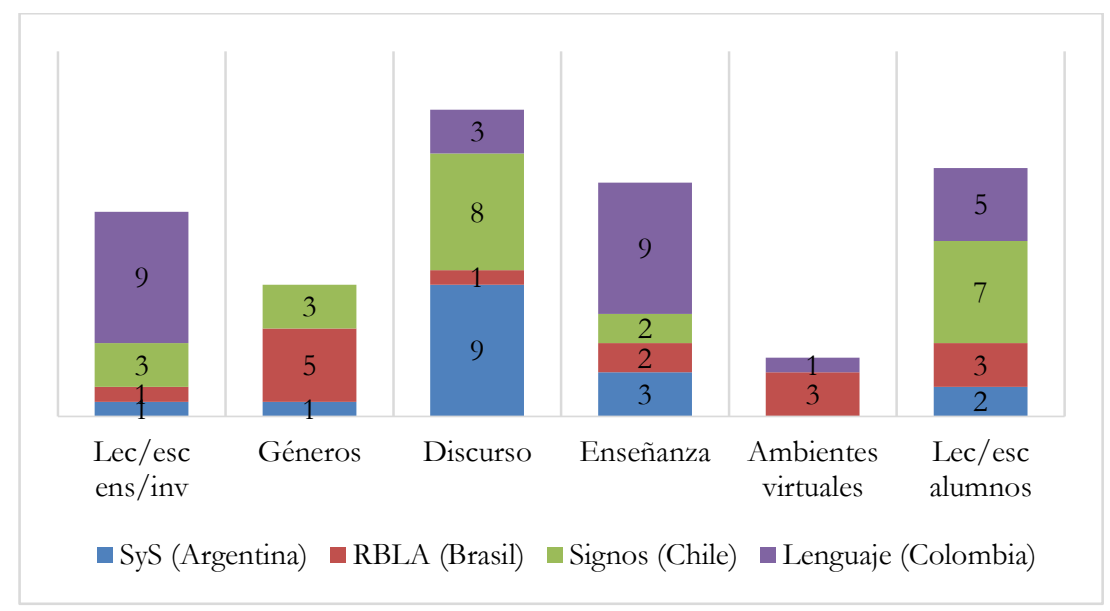

Gráfico 5. Cantidad de artículos por tópico y revista.

Los temas que generan más interés en la región son el discurso (n. 21; 25,9\%), la lectura y escritura estudiantiles (n. 17; 21\%) y la enseñanza (n. 16; 19,7\%). Estos datos muestran, por un lado, la importancia del análisis de fenómenos textuales propios del 
discurso científico y académico y, por el otro, el interés por las prácticas letradas estudiantiles y su enseñanza. Al distinguir entre países, se verifica que los estudios del discurso son prioritarios en las revistas chilena y argentina, pero escasos en las revistas brasileña y colombiana. Además, en el corpus de Brasil se encuentran consolidados los géneros discursivos y/o textuales como objeto de estudio, mientras que en el corpus de Colombia se prioriza la enseñanza e investigación de la lectura y la escritura.

Además de los temas, en la codificación se determinaron 13 orientaciones teóricas predominantes explícitas en las que se encuadran los artículos del corpus. Estas son:

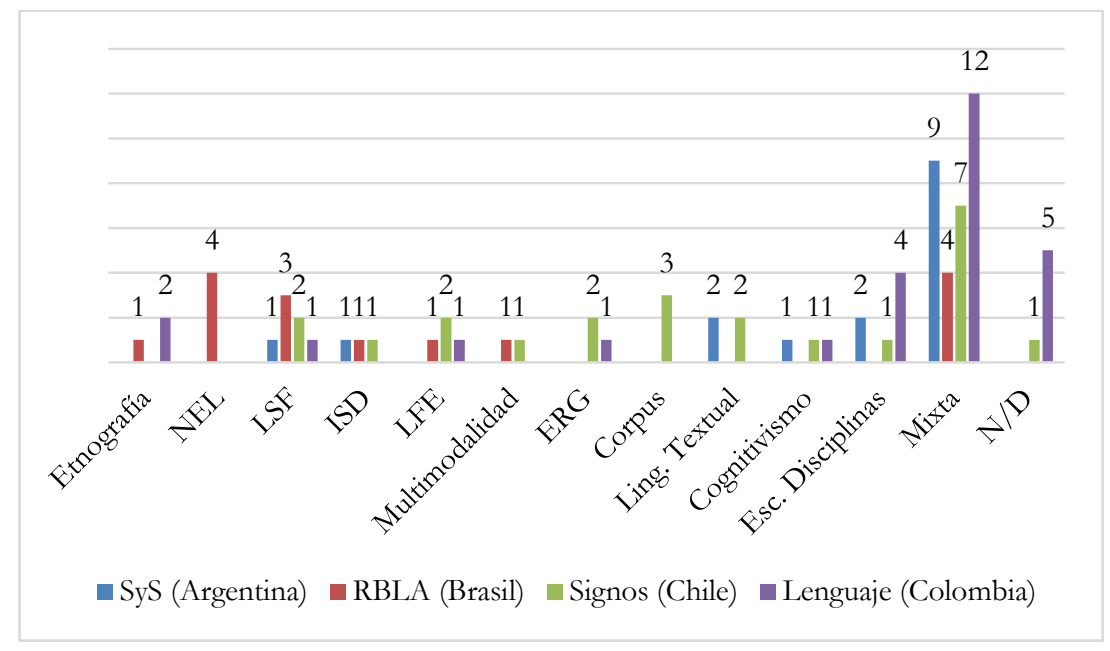

Gráfico 6. Cantidad de artículos por orientación y revista.

El Gráfico 6 muestra la predominancia de las orientaciones mixtas (n. 32; 39,5\%), una de nuestras hipótesis de trabajo: el espacio disciplinar se caracteriza por yuxtaponer o articular tradiciones y marcos teóricos diversos (por ejemplo, lenguas para fines específicos, lingüística sistémico-funcional, escritura en las disciplinas y lingüística textual) que no son cercanos en sus orígenes; este patrón es especialmente marcado en las revistas colombiana y argentina. Ninguna tendencia teórica muestra un predominio dentro del corpus total, aunque se destacan la Lingüística SistémicoFuncional y la Escritura en las Disciplinas (ambas con n. 7; 8,6\%). Los artículos de Lenguaje se destacan por su eclecticismo, junto con el interés por inscribirse en la línea de Escritura en las Disciplinas, fuertemente asociada a los trabajos de Paula Carlino. En RBLA es predominante la línea de Nuevos Estudios de Literacidad y de Lingüística Sistémico Funcional. Revista Signos. Estudios de Lingüística se nutre de tradiciones diversas pero es la única revista que se inclina por la Lingüística de Corpus, en particular desde las investigaciones de la Escuela Lingüística de Valparaíso.

El análisis histórico de orientaciones teóricas también permite detectar dinámicas de emergencia y declive, tal como ilustra el siguiente Gráfico, que agrupa los artículos en lustros: 


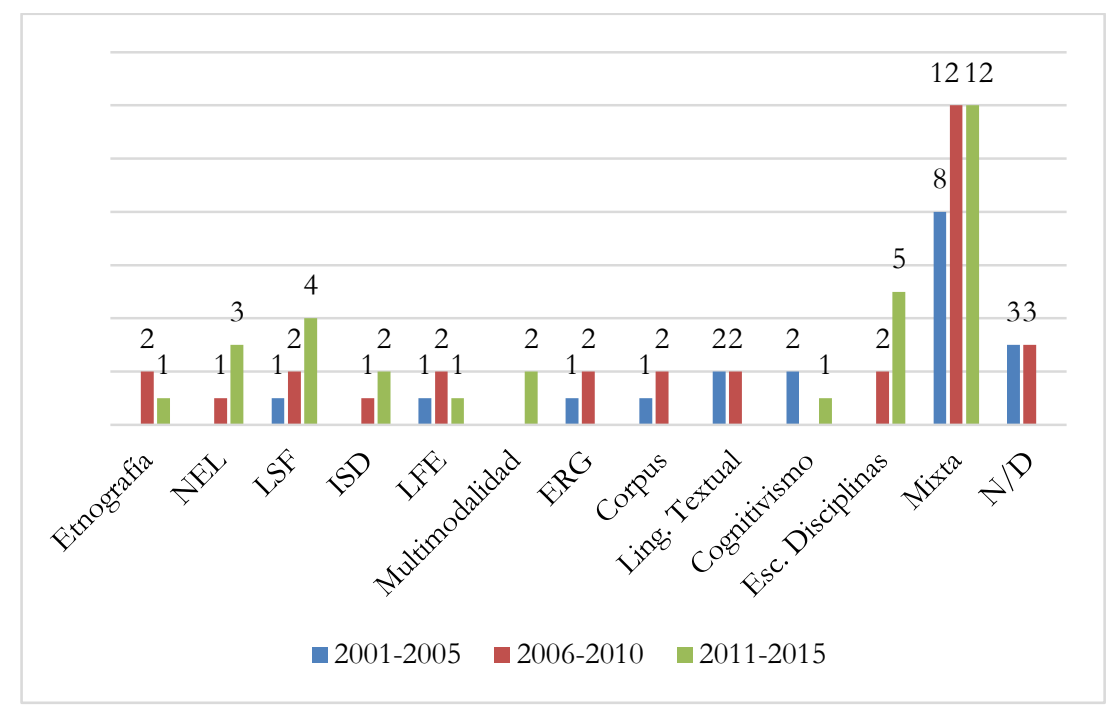

Gráfico 7. Cantidad de artículos por orientación y lustro.

El Gráfico 7 histórico muestra cambios interesantes en las orientaciones. Por un lado, la desaparición de los artículos con una orientación no declarada en el último lustro, lo cual insinúa un incremento de la presencia explícita de los marcos teóricos en los que se insertan las investigaciones. Por el otro, la emergencia del marco de Escritura en las Disciplinas (en particular en el corpus colombiano, como se ve en el Gráfico 6) y, en menor medida, Lingüística Sistémico-Funcional, Nuevos Estudios de Literacidad (en Brasil), Interaccionismo Socio-Discursivo y Multimodalidad, así como la reducción de la Lingüística de Corpus y la Lingüística Textual.

La variable nivel o entorno educativo abordado por los artículos también arrojó resultados de interés:

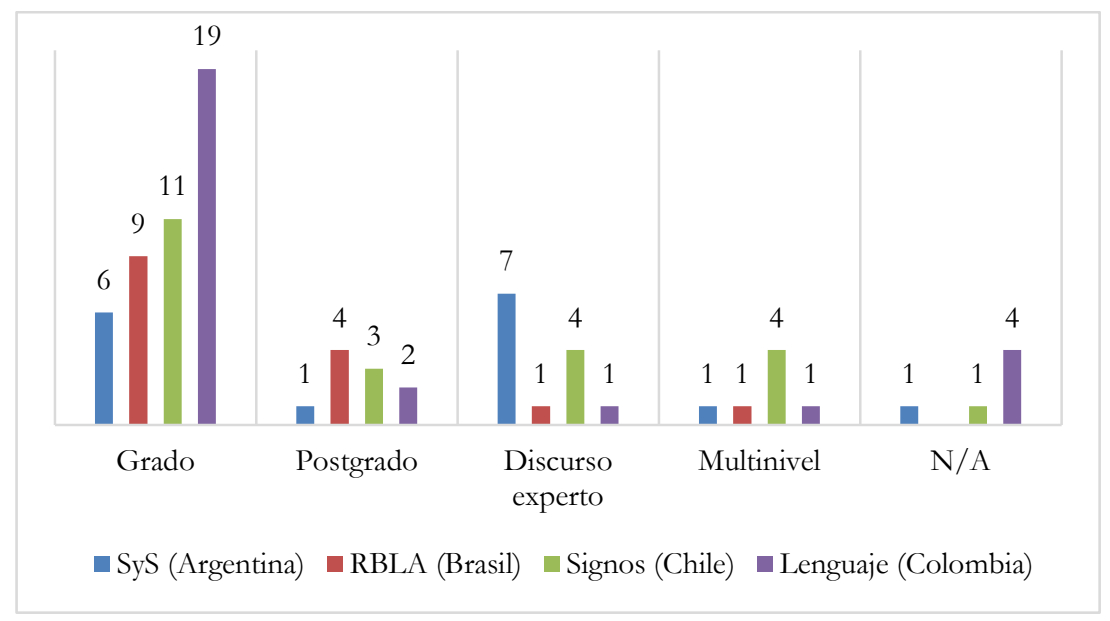

Gráfico 8. Cantidad de artículos por nivel y revista. 
El Gráfico 8 muestra una predominancia clara de los estudios acerca del grado universitario (n. 45; 55,6\%) en la región, así como un menor interés por la lectura y la escritura de posgrado (n. 10;12,3\%). Al distinguir entre países, el corpus de Colombia se interesa casi exclusivamente por el grado, el corpus de Argentina muestra un marcado interés por el discurso experto, el corpus de Brasil por el discurso de grado y posgrado y el corpus de Chile balancea su interés por los diferentes niveles educativos. Cabe agregar que los estudios sobre el posgrado muestran un crecimiento histórico (datos no incluidos en el Gráfico 8): ninguno en el primer lustro considerado, 4 en el segundo lustro y 6 en el último.

El Gráfico 9 compara las áreas disciplinares investigadas por los artículos:

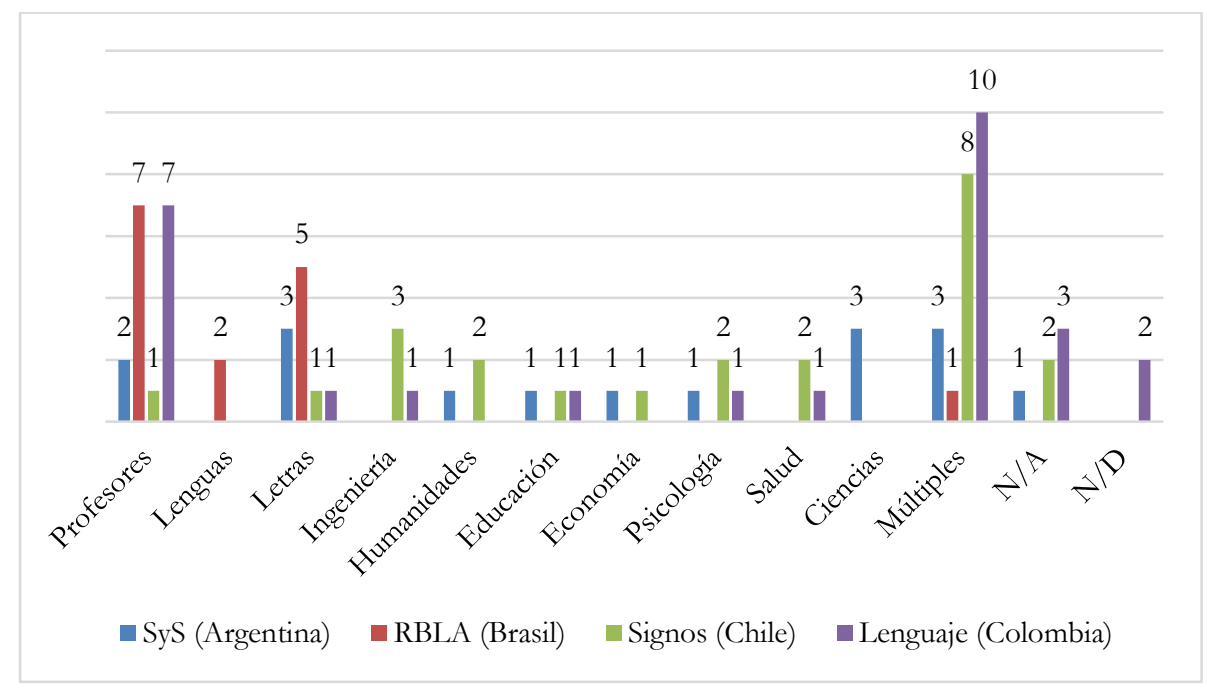

Gráfico 9. Cantidad de artículos por área y revista.

A nivel regional, predomina el interés por estudiar áreas disciplinares diversas en una misma investigación (n. 22; 27,2\%), en especial en Chile y Colombia, o por la formación de profesores (n. 17; 21\%), sobre todo en Brasil y Colombia. En contraste, Argentina no muestra preferencias por ningún área en particular. Los artículos de Signos abordan más áreas distintas, mientras que los artículos de RBLA se concentran en menos áreas. Los resultados también demuestran que la mayoría (n. 41; 50,6\%) de áreas específicas estudiadas pertenecen a las Humanidades y las Ciencias Sociales (incluida Psicología).

Por otro lado, también es relevante indagar sobre los géneros discursivos y/o textuales de interés: 


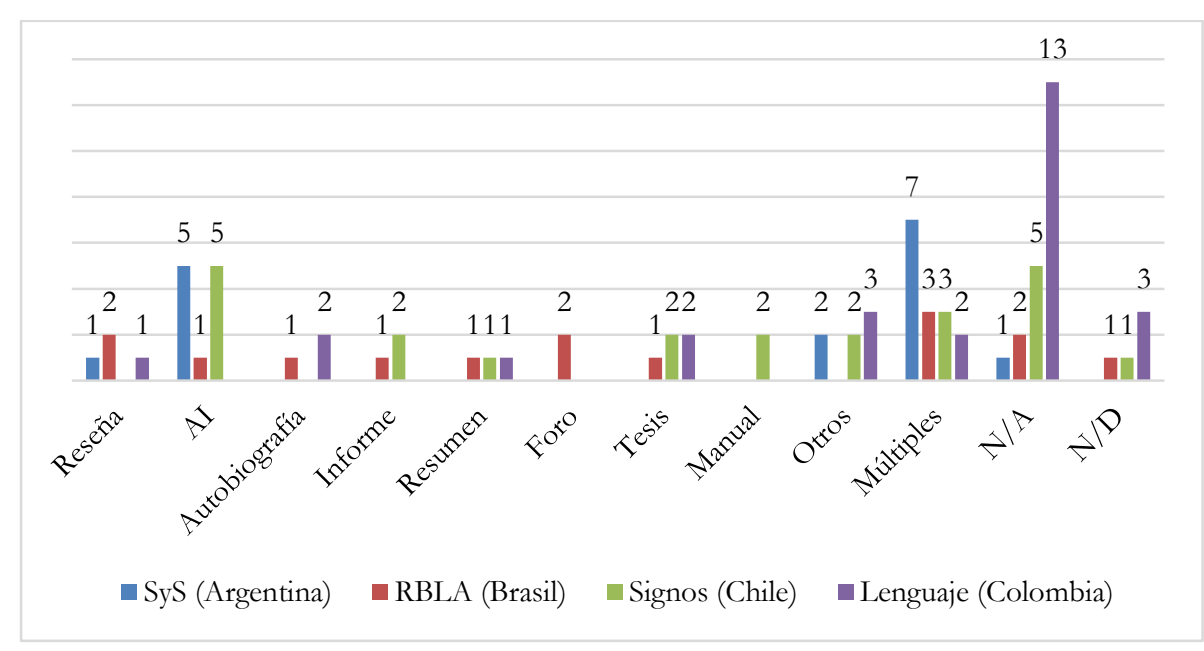

Gráfico 10. Cantidad de artículos por género y revista.

Si se descartan las investigaciones en las que no aplica la categoría o en las que no es posible especificar qué géneros son investigados, las tendencias predominantes muestran un interés por investigar más de un género discursivo y/o textual a la vez (n. $15 ; 18,5 \%)$, en especial en la revista argentina, o por el artículo de investigación científica (n. 11; 13,6\%), sobre todo en el corpus chileno y argentino. Aparecen otros géneros frecuentes como la tesis de grado y postgrado (n. 5; 6,2\%), la reseña (n. 4; $4,9 \%$ ) y la autobiografía, el informe y el resumen (n. 3; 3,7\%). El contraste regional muestra que en Colombia son frecuentes las investigaciones sobre prácticas de lectura y escritura independientemente de los géneros involucrados y que Brasil se destaca por un interés por una gran diversidad de géneros.

Los países que integran los corpora se especializan en los tipos de artículos que publican:

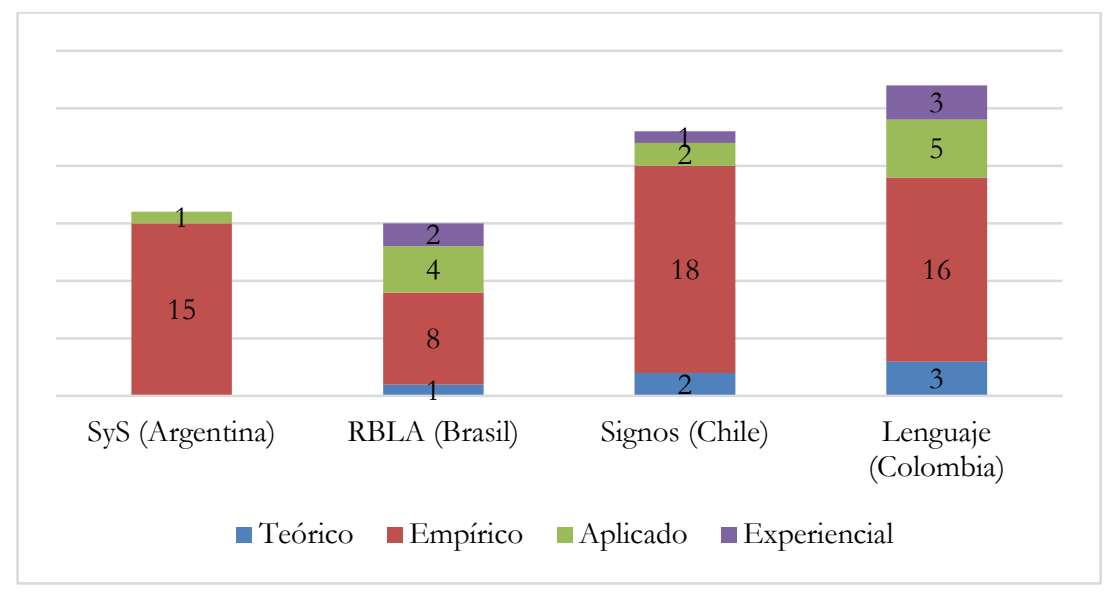

Gráfico 11. Cantidad de artículos por revista y tipo. 
El corpus argentino es el único que no incluye investigaciones teóricas ni experienciales y que, por el contrario, se especializa en investigaciones de base empírica, en una línea similar a la chilena. En contraste, el corpus brasileño muestra mayor balance entre los estudios empíricos y los estudios de los demás tipos, en una tendencia similar a la colombiana. En términos regionales, debe destacarse la predominancia de investigaciones empíricas (n. 57; 70,4\%) y la escasa aparición de teorizaciones y relatos de experiencias sin datos de base (n. 6; 7,4\%, cada uno).

\section{CONCLUSIONES}

Dado que los corpora de análisis están vinculados a una sola publicación científica por cada país, por influyente que esta sea, es probable que algunos de los resultados no representen tendencias nacionales sino preferencias de la publicación, como su alcance temático o la cultura disciplinar de la institución de pertenencia. Por otro lado, la selección de revistas dejó de lado países de peso en el área, como México y Venezuela. Estudios futuros deberían ampliar el corpus a otras revistas influyentes de estos u otros países de la región de forma tal de confirmar, complejizar o desafiar nuestros resultados. A pesar de estas salvedades metodológicas, los resultados presentan tendencias sugerentes, confirman hallazgos previos y permiten respaldar empíricamente hipótesis sobre la configuración de este espacio disciplinar.

Primero, los resultados muestran una mayoría de artículos interesados en la investigación empírica de la escritura. Se abordan entornos y géneros diversos, aunque con predominancia en formación de grado y el área de las Humanidades y las Ciencias Sociales y con foco en el artículo de investigación científica. Están escritos por investigadores de países diversos y en su mayoría se publican en español y portugués.

Segundo, se comprueba un marco generalizado de orientaciones teóricas, tradiciones y metodologías generales sumamente diversas y heterogéneas; estos hallazgos refuerzan y profundizan investigaciones recientes del equipo ILEES (Bazerman et al., en prensa). En efecto, la complejidad de la escritura como objeto de reflexión invita al eclecticismo teórico y a la integración de datos desde múltiples perspectivas disciplinares (Bazerman, 2011). A su vez, el análisis histórico muestra un incremento reciente de los marcos teóricos explícitos y la aparición de algunas orientaciones novedosas.

Tercero, en el corpus colombiano se detecta un interés por la lectura y escritura como prácticas y procesos situados que se investigan desde una perspectiva educativa, mientras que los corpora argentino y chileno se destacan por una perspectiva lingüística sobre la lectura y escritura como discursos especializados o productos de esos discursos; el corpus brasileño se ubica en un punto intermedio en este continuo.

Cuarto, los corpora brasileño y colombiano muestran dinámicas de intercambio más bien locales, en contraste con los corpora argentino y chileno que muestran una 
participación mayor de investigadores regionales y extranjeros. Ávila Reyes (2015) llega a conclusiones similares desde una perspectiva y corpus diferentes. El corpus brasileño es un caso particularmente interesante: los resultados sugieren un campo científico amplio y profesionalizado, donde es poco frecuente publicar en la institución de pertenencia, pero al mismo tiempo con escasos vínculos académicos con el resto del continente. Estos resultados también confirman investigaciones previas del equipo ILEES.

Por último, en cuanto a la perspectiva histórica, hay diversas tendencias que vale destacar a lo largo del periodo analizado, pese a su brevedad. El primero es que las publicaciones sobre el tema comienzan recién a aparecer en 2003, de modo concomitante en Chile y Colombia; y su número fluctúa en el tiempo, con máximos de 11 artículos en total, potenciados por la aparición de números especiales sobre el tema. Otra interesante constatación tiene que ver con el énfasis en la escritura por sobre la lectura: si bien la escritura predomina en general, se constata una pequeña relevancia de la lectura en los años iniciales y un notable descenso a partir de 2008, probablemente debido al aumento desde entonces del foco en la lectura y escritura como fenómenos interdependiente, es decir, un menor foco en la lectura como competencia aislada.

Con respecto a las orientaciones teóricas, al agrupar por lustros, se observa que presentan también dinámicas que permiten inferir la emergencia del espacio disciplinar de la escritura universitaria: primero, en el período 2006-2010 aparece la orientación de 'escritura en las disciplinas' como marco claramente definido, el que aumenta en el lustro siguiente en tres artículos (2011-2015). Segundo, los artículos con orientaciones teóricas sin declarar descienden a cero en el último período, lo que sugiere una mayor reflexión y claridad de los marcos epistemológicos para abordar este tema. Tercero, los artículos con orientaciones teóricas mixtas aumentan en el segundo y tercer lustros (2006 a 2015). Como se ha planteado antes, la hibridación y mixtura es una característica de las disciplinas emergentes (Klein, 1996), lo que puede orientar la interpretación de estos patrones de tendencias teóricas como una etapa de maduración del campo. Un último resultado de carácter histórico lo constituye el aumento progresivo por estudiar la escritura en el contexto del postgrado, cuyas causas pueden radicar tanto en la expansión natural del objeto de estudio, como en el aumento de la matrícula y los programas en este nivel.

En suma, hemos identificado algunos de los elementos que constituyen las prácticas compartidas disciplinarmente en un marco de incremento de los anclajes institucionales y burocráticos (congresos, posgrados, asociaciones) que aseguran su continuidad y expansión en la región, pero a la vez hemos encontrado préstamos e hibridizaciones teóricas que brindan permeabilidad a las fronteras disciplinares, así como dinámicas y preferencias netamente locales. Estos resultados permiten continuar 
avanzando en la delimitación explícita de un espacio disciplinar, con al menos dos décadas de desarrollo, interesado en la investigación de la lectura y escritura en educación superior en América Latina. Esta empresa resulta de importancia científica, educativa y política para fomentar su curricularización en la formación de grado y posgrado, para favorecer el financiamiento de becas y proyectos de investigación (Phelps \& Ackermann, 2010), para legitimar su especificidad en la creación de cargos de docencia y gestión, para generar consensos (o formas consensuadas de debatir) respecto de las metodologías posibles y los marcos teóricos existentes y por desarrollar, para multiplicar los espacios e instancias de intercambio y discusión, por ejemplo en revistas y congresos, para incentivar las formas de comunicación propias y para reflexionar sobre su historia y denominación y fomentar caminos de desarrollo futuro. 


\section{REFERENCIAS BIBLIOGRÁFICAS}

Ávila Reyes, N. (2015). Intertextual dynamics of an emerging field: An exploration of citation practices of writing studies in six Latin American countries (20022014). Tesis de Maestría, Universidad de California, Santa Bárbara, Estados Unidos.

Ávila Reyes, N., González-Álvarez, P. \& Peñaloza Castillo, C. (2013). Creación de un programa de escritura en una universidad chilena: Estrategias para promover un cambio institucional. Revista Mexicana de Investigación Educativa, 18(57), 537560.

Bazerman, C. (2011). The disciplined interdisciplinarity of writing studies. Research in the Teaching of English, 46(1), 8-21.

Bazerman, C., Ávila Reyes, N., Bork, A. V., Corrêa, F., Cristovão, V. L., Tapia-Ladino, M. \& Narváez, E. (en prensa). Intellectual orientations of studies of higher education writing in Latin America. En S. Plane, C. Bazerman, C. Donahue \& F. Rondelli (Eds.), Recherches en écriture: Regards pluriels. Nancy: Université de Lorraine.

Becher, T. (2001). Tribus y territorios académicos. La indagación intelectual y las culturas de las disciplinas. Barcelona: Gedisa Editorial.

Bork, A. V., Bazerman, C., Correa, F. \& Cristovão, V. L. (2014). Mapeamento das iniciativas de escrita em língua materna na educação superior: Resultados preliminares. Revista Prolíngua, 9(1), 2-14.

Bourdieu, P. (2003). Los usos sociales de la ciencia. Buenos Aires: Nueva Visión.

Carlino, P. (2003). Alfabetización académica. Un cambio necesario, algunas alternativas posibles. Educere. Investigación, 6(20), 409-417.

Carrasco, A., Encinas, F., Castro, M. C. \& López Bonilla, G. (Eds.) (2013). Lectura y escritura académica en la educación media superior y superior. Revista Mexicana de Investigación Educativa, 18.

Cubo de Severino, L. (Ed.) (2005). Comunicación académico-científica. Signo y Seña, 14.

Ezcurra, A. M. (2011). Abandono estudiantil en educación superior. Hipótesis y conceptos. En N. Gluz (Ed.), Admisión a la universidady selectividad social. Cuando la democratización es más que un problema de "ingresos" (pp. 23-62). Los Polvorines: Universidad Nacional de General Sarmiento. 
Fernández Lamarra, N. \& Costa de Paula, M. (Eds.) (2011). La democratización de la educación superior en América Latina. Limites y posibilidades. Tres de Febrero: EDUNTREF.

Goggin, M. D. (2000). Authoring a discipline. Scholarly journals and the Post-World War II Emergence of Rhetoric and Composition. Mahwah, NJ: Lawrence Erlbaum.

Gold, D. (2012). Remapping revisionist Historiography. College Composition and Communication, 64(1), 15-34.

Gläser, J. \& Laudel, G. (2013). Life with and without coding: Two methods for early stage data analysis in qualitative research aiming at causal explanations. Forum: Qualitative Social Research, 14(2), 1-25.

Halliday, M. A. K. (2004). On the Language of Physical Science. En J. Webster (Ed.), The language of Science (pp. 140-158). Londres: Continuum.

Haswell, R. H. (2005). NCTE/CCCC's recent war on scholarship. Written Communication, 22(2), 198-223.

Hawk, B. (2007). A counter-History of composition: Toward methodologies of complexity. Pittsburgh, PA: University of Pittsburgh Press.

Hyland, K. (2011). Disciplines and discourses: Social interactions in the construction of knowledge. En D. Starke-Meyerring, A. Paré, N. Artemeva, M. Horne \& L. Yousoubova (Eds.), Writing in Knowledge Societies (pp. 193-214). Anderson, South Carolina: The WAC Clearinghouse-Parlor Press.

Jacobs, J. (2013). In defense of disciplines: Interdisciplinarity and specialization in the research university. Chicago: University of Chicago Press.

Klein, J. T. (1996). Crossing boundaries: Knowledge, disciplinarities, and interdisciplinarities. Charlottesville: University of Virginia Press.

Machado, A. R., Lousada, E. G. \& Tardelli, L. A. (2005). Planejar gêneros acadêmicos. São Paulo: Parábola.

Miller, T. (2010). The evolution of College English: Literacy studies from the Puritans to the Postmoderns. Pittsburgh, PA.: University of Pittsburgh Press.

Motta-Roth, D. (2014). ONE account on the current research landscape on academic Literacy practices in Brazil. Ponencia presentada en III Writing Research Across Borders (WRAB) International Conference, París, Francia.

Mueller, D. (2012). Grasping rhetoric and composition by its long tail : What graphs can tell us about the field's changing shape. College Composition and Communication, 64(1), 195-224. 
Narváez Cardona, E. (2012). Training Experiences in Reading and Writing in a Colombian University: The Perspective of a Professor. En C. Thaiss, G. Bräuer, P. Carlino, L. Ganobcsik-Williams \& A. Sinha (Eds.), Writing Programs Worldwide: Profiles of Academic Writing in Many Places (pp. 147-156). West Lafayette, Indiana: Parlor Press \& WAC Clearinghouse.

Navarro, F. (2012). Alfabetización avanzada en la Argentina. Puntos de contacto con la enseñanza-aprendizaje de L2. Revista Nebrija de Lingüistica Aplicada a la Enseñanza de las Lenguas, 12(6), 49-83.

Ortega de Hocevar, S. (2015). Cátedra UNESCO Argentina: Acciones académicas, líneas de investigación; vinculación con el medio y la universidad. En C. Muse (Ed.), Lectura y escritura 11: Lectura y escritura. Algunas miradas desde América Latina (pp. 50-57). Córdoba: Universidad Nacional de Córdoba.

Ortiz Casallas, E. M. (2011). La escritura académica universitaria: Estado del arte. Íkala, Revista de Lenguaje y Cultura, 16(28), 17-41.

Parodi, G. (Ed.). (2010). Alfabetización académica y profesional en el siglo XXI. Leer y escribir desde las disciplinas. Santiago de Chile: Ariel.

Pereira, C. (2006). La lectura y la escritura en el CBC: Memoria de la experiencia en la cátedra de Semiología. Ponencia presentada en el Primer Congreso Nacional: "Leer, escribir y hablar hoy". Universidad Nacional del Centro de la Provincia de Buenos Aires, Argentina.

Phelps, L. W. \& Ackerman, J. (2010). Making the Case for Disciplinarity in Rhetoric, Composition, and Writing Studies: The Visibility Project. College Composition and Communication, 62, 180-215.

Russell, D. (2006). Historical studies of composition. En P. Smagorinsky (Ed.), Research on Composition: Multiple Perspectives on Two Decades of Change (pp. 217273). Nueva York: Teachers College.

Tapia-Ladino, M. (2013). Lectura y escritura en Educación Superior en publicaciones de la Revista Signos. Ponencia presentada en XII Congreso Latinoamericano para el Desarrollo de la Lectura y la Escritura y IV Foro Iberoamericano de Literacidad y Aprendizaje, Puebla, México.

Thaiss, C., Bräuer, G., Carlino, P., Ganobcsik-Williams, L. \& Sinha, A. (2012). Writing Programs Worldwide: Profiles of Academic Writing in Many Places. Parlor Press \& The WAC Clearinghouse.

UNESCO. (2009). Compendio mundial de la educación. Montral: UNESCO-UNIS. 
Venegas, R., Núñez, M. T., Zamora, S. \& Santana, A. (2015). Escribir desde la Pedagogía del Género. Guías para escribir el Trabajo Final de Grado en Licenciatura. Valparaíso: PUCV.

\section{NOTAS}

${ }^{1}$ Investigación parcialmente financiada por la UCSB Academic Senate Research Grant (2012-2013); la Conference on College Composition and Communication Research Initiative (2012-2014); el Programa de Becas de Posgrado para Investigadores del Ministerio de Educación de la Nación Argentina y la Comisión Fulbright (2015-2016); el Proyecto Basal FB0003 del Programa de Investigación asociativa de CONICYT; y el Plan de Mejoramiento Institucional (PMI) UCH1501 del Ministerio de Educación, Chile.

${ }^{2}$ http://www.lecturayvida.fahce.unlp.edu.ar/; consultado el 16/01/2015.

${ }^{3}$ http://siget2015.fflch.usp.br/apresentacao; consultado el 16/01/2015.

${ }^{4}$ http://ascun.org.co/red/detalle/red-de-lectura-y-escritura-en-educacion-superior-redless; consultado el $11 / 01 / 2015$.

${ }^{5}$ https://sites.google.com/site/redlacpe/; consultado el 16/01/2015.

${ }^{6}$ http://www.utp.edu.co/vicerrectoria/investigaciones/publicaciones-lectura-escritura/; consultado el $16 / 01 / 2015$.

${ }^{7}$ http://revistas.filo.uba.ar/index.php/sys/about; consultado el 07/01/2015.

${ }^{8}$ http://www.scielo.br/revistas/rbla/eaboutj.htm; consultado el 07/01/2015.

${ }^{9}$ http://www.scielo.cl/revistas/signos/eaboutj.htm; consultado el 07/01/2015.

10 http:// revistalenguaje.univalle.edu.co/index.php/Lenguaje/about/editorialPolicies\#focus AndScope; consultado el 07/01/2015. 